\title{
GÊNES ET L'AFRIQUE DU NORD VERS 1450: LES VOYAGES «PER COSTERIAM»
}

Tous les ports chrétiens de la Méditerranée occidentale, de Provence, de Catalogne et d'Italie surtout, entretiennent malgré toutes sortes de difficultés, malgré la piraterie plus ou moins chronique et le cycle souvent drametique des représailes, d'étroits rapports marchands avec les royaumes musulmans du Maghreb. Pour certains hommes d'affaires, c'est même là un champ d'action priivilégié. Il y vendent quantité de riches draps anglais, des étoffes catalanes, de la vaisselle de cuivre et de l'or. Au $\mathrm{XV}^{\mathrm{eme}}$ siècle, le royaume de Tunis s'affirme, avec la Sicile, l'un des grands greniers du monde méditerranéen, exportateur chaque année '. Ce même royaume afferme à très hauts prix, régulièrement à des sociétés génoises, le droit pêcher le corail sur le littoral de Marcasarès (Mers-el-Kharez) ${ }^{2}$ et d'installer des madragues pour le thon dans la baie de Sousse ${ }^{3}$. Dans tous ces ports du Maghreb, se négocie l'or du Soudan, l'or tiberi, source de grands profits, principal moteur parfois de ces négoces ${ }^{4}$.

Gênes, dont la vocation «occidentale» et nord-africaine s'est imposée très tôt, dès les premiers moments de la Reconquête chrétienne et ensuite lors des luttes contre les Sarrazins, prend naturellement une part très active à ces échanges. Ses navires se présentent dans les ports, ses marchands s'y établissent volontiers, du moins les plus aventureux d'entre eux ou leurs commis; à Tunis et à Sousse, ils ont leur fondouk particulier, ils vont, dans l'intérieur, acheter des grains à Constantine'.

' R. BRUNSCHVING, La Berbérie orientale, Paris, 1947; J. HeERS, Gênes au XV'm siècle; activités économiques et problèmes sociaux, Paris, 1961, pp. 329 et sq.

2 Ibid, pp. 426-428. F. PODESTA, La pesca del corallo in Africa nel Medioevo; i Genovesi a Marsacares, Gênes, 1897.

' J. HeERS et G. DE GROER, L'itinéraire d'Anselme Adorno en Terre Sainte (14701471), Paris, 1974, pp. 144-147; des thons si nombreux que notre voyageur prétend que la ville de Tunis «tire son nom de ce poisson»; pris d'hésitation, il ajoute cependant: «à moins que le poisson, que les Maures appellent ton (?) n'eût tiré son nom de la ville».

${ }^{4}$ par ex.: A.E. SAYOUS, Le commerce des Européens à Tunis depuis le XII'm siécle jusqu'à la fin du XViu' siècle, Paris, 1929.

' J. HeERS, Gênes, (cité supra) p. 476. 
Cependant ces négoces génois, ne se limitent généralement pas à de simples achats de produits bruts aussitôt expédiés vers l'Italie. Cette action se définit plus complexe que, par exemple, celle des gens de Marseille ou de Barcelone. De nombreux navires de la cité ligure touchent, en un seul voyage, tous les ports, d'Honein à Tripoli et, souvent, leur patron s'attarde, visite les escales les unes après les autres, longent de près le littoral et poursuivent leur voyage, non vers Gênes, mais directement vers l'Orient ou vers l'Atlantique. En effet, la grande originalité du système de navigation génois, réside en ce fait qu'une part fort importante du trafic assuré par ses bâtiments ne passe pas par le port de Gênes; mais, au contraire, joint directement le Levant méditerranéen à l'Occident lointain (Flandre et Angleterre) en longeant la côte sud de la mer intérieure. Ces grandes et laborieuses entreprises maritimes, qui peuvent durer plusieurs semaines et même toute une saison, dont on ne rencontre aucun équivalent chez d'autres nations, deviennent une véritable routine et obéissent à des usages bien établis: c'est aller "per costeriam».

Cette expression "per costeriam», typiquement et même exclusivement génoise, est dans la ville d'un emploi très courant; chaucun s'y réfere par habitude bien ancrée: les notaires pour rédiger les contrats d'affrêtements, les témoins appelés à parler de tel ou tel accident, les scribes des douanes, les patrons de navires ou leurs commis pour consigner les opérations à bord. Et, d'autre part, "per costeriam» ne s'applique strictement qu'aux navigations au long des côtes du Maghreb; jamais pour celles en Grêce, ou en Asie mineure, ou en quelque région que ce soit en Orient; pas même pour des pays qui, a priori, présenteraient les mêmes difficultés de contacts, côtes occidentales de la Mer Noire dans les régions de Valachie et de Transylvanie, côtes orientales pour le Caucase.

L'organisation de tels transports maritimes, pour ces nefs de Gênes d'un tonnage énorme, peu maniables donc, maladroites et dangereuses précisément aux approches du littoral, et qui, de plus, coûtent très cher à chaque perte de temps, cette organisation répondait forcément à d'impérieuses sollicitations.

Sans doute pourrait-on invoquer des avantatges fiscaux, la possibilité d'échapper aux taxes portuaires à Gênes même, et la facilité de frauder pour ces trafics dits "de transit» ou d'obtenir divers arrangements; les patrons de navires ne s'en provent pas et, dès lors qu'ils s'engagent à déclarer toutes leurs cargaisons, se voient bénéficier d'importants réductions de taxes ${ }^{6}$. En outre, pour certains commerces, ces voyages "per costeriam» représentent une grande économie de distance, dans la mesure où, pour divers produits, Gênes et son

- Archivio di Stato di Genova (cité dès lors: ASG); Archivio di San Giorgio, Carati Maris 1445 , sala 38/55, n. ${ }^{\circ} 1616$ (copie d'un grand nombre de contrats pour réductions de taxes). 
arrière-pays ne constituent pas d'importants marchés et, en aucun cas, des étapes indispensables. Ainsi, par exemple, pour les nefs qui assurent les liaisons entre le Nord-Ouest européen, régions de fortes industries drapantes, et l'Orient méditerranéen, gros producteur, jusqu'en 1456-1458, de l'alun, mordant pour la teinture des tissus. Ces échanges, qui ne font pas intervenir le port de Gênes, s'effectuent pourtant sur une très vaste échelle. Il s' agit, au plein sens du terme, d'une affaire vraîment internationale et, de plus, d'une forte présence économique dans le monde musulman du Maghreb ou, pour le moins, sur ses rivages. Le patron, ses marins, les marchands qui se trouvent à bord, ne se contenten pas de rares points d'escales pour y rencontrer leurs seuls nationaux, frères ou cousins, associés ou commis, cantonnés dans un caravansérail. Bien au contraire, ils cherchent divers contacts, en toutes occasions; ils prospectent des marchés; d'où une insertion plus intense que celle que l'on imaginerait à ne penser qu'aux heurts de civilisations, aux hostilités et aux représailles. Non pas du tout une «exploitation» limitée à quelques comptoirs et forcément superficielle, mais une recherche plus en profondeur; une situation qui, toutes circonstances ponctuelles mises à part bien sûr, rappelle celle que connaissent depuis plus de deux siècles Vénitiens et Génois sur le côtes de l'empire byzantin, sur les plages et aux foires du Péloponnèse, dans des positions parfois très aventurées?

Autre facteur à ne pas négliger dans cette analyse des contacts: le fait d'effectuer des transports pour le compte des marchands musulmans du royaume de Grenade, de Malaga surtout, et de tous les ports importants jusqu'à Tripoli et Alexandrie; nombre d'entre eux ne quittent le bâtiment génois qui les a pris à son bord qu'en Asie Mineure ou à Chio, île génoise. Sans doute pouvons-nous reconnaître, avec Charles-Emmanuel Dufourcq, que ces marins musulmans ne se limitaient pas exclusivement au petit cabotage ou à la piraterie, mais lançaient aussi des navires plus importants ${ }^{8}$. Leurs flottes marchandes n'avaient pas complètement disparu de la surface des mers et pouvaient toujours rendre de grands services. Mais, cela étant assuré, il reste que les navires de Gênes, à Malaga, à Honein ou à Oran, à Tunis, chargeaient très régulièrement de nombreux marchands «maures» et des quantités de produits sous leurs noms, voire même des esclaves les accompagnant. Ces

' Voir par exemple: G.M. ThOMAS et T. PRedelli, Diplomatarium VenetoLevantinum, sive Acta et diplomata res Venetas Graecas atque Levantis illustrentia (13001454), 2 vol., Venise, 1880-1899 (Monumenti storici pubblicati dalla Reale Deputazione veneta di Storia Patria. Documenti, V).

${ }^{8} \mathrm{CH}$. E. DUFOURCQ, Commerce du Maghreb médiéval avec l'Europe chrétienne et marine musulmane: données connues et problèmes en suspens, in «Actes du Congrès d'Histoire de la Civilisation du Maghreb», Tunis, 1979, pp. 161-192. 
transports de «passagers» ne laissent malheuresement que peu de traces chez les notaires pourtant si bavards sur tant de points.

Quelques contrats en témoignent pourtant.

En 1456, un associé réclame à Gênes les comptes assez complexes d'un voyage d'Afrique et, en particulier, 260 ducats d'or, frêt de marchandises chargées à bord par des «maures» d'Alexandrie ${ }^{9}$. Bien plus intéressant que cette simple mention noyée dans un fatras comptable, s'avère un "compromissum» signé à Chio, le 29 janvier 1459, entre, d'une part, les deux patrons d'un navire qui vient de s'ancrer dans le port, Maurizio Cattaneo et Thobie Lomellino, et, d'autre part, plusieurs musulmans (infrascripta mauri mercatores et habentem raubam et merces in ipsa nave): neuf hommes de Tripoli, cinq de Tunis, deux de Grenade et deux autres de Fasi (?). Ces marchands-passagers contestent les sommes exigées pour leurs frêts et, après de longues palabres plus l'intervention d'un expert, il est entendu qu' ils devront payer quatre ducats et trois quarts de ducat de Chio pour chaque sac de lin transporté d'Alexandrie à Chio; pour les autres produits, l'on diminue également le taux du frêt par de savants calculs ${ }^{10}$.

Les choses apparemment ne vont pas toutes seules... mais elles s'arrangent tout de même car, pour rentrer chez eux, il leur faut passer par les Génois. À preuve un autre contrat, d'affrêtement celui-ci, signé deux jours plus tard, le 31 janvier, par ces mêmes marchands "maures» auxquels s'est joint un homme originaire de Syrie. Leonardo de Maris, patron d'une grosse nef génoise de 14.000 cantares de portée ( 660 tonnes métriques), déclare charger à son bord les cinq cents sacs de lin qui leur restent, mais il entend aller d'abord à Phocée, sur la côte d'Anatolie, où il doit rester très longtemps, 46 jours entiers; de là, il gagnera Tunis où il déchargera ces sacs. Cette fois, le frêt est fixé à l'avance, plus faible que pour le précédent voyage pourtant plus court: $31 / 4$ ducats de Chio par sac. Si le mauvais temps l'oblige à s'arrêter à Sousse, ils ne paieront que deux ducats per sac; et pour Tripoli, seulement un ducat et demi. Ce frêt est à payer dans les dix jours qui suivent le débarquement; mais ils ne déchargeront, dans un premier temps, que deux cents sacs et les autres seulement après un premier paiement. Le patron leur réserve six belles "chambres» de son navire; il leur fournit l'eau et le bois pour se chauffer; il ne leur fait rien payer pour leurs passages, ni pour leurs domestiques ou commis (ac eius famulos), ni pour leurs flacons ou tonnelets d'eau de rose et leurs sacs de voyage, ni pour leur pacotille. Il leur avance même l'argent qu'ils doivent encore aux

9 ASG, Notaio Tommaso Duracino, filza 2, parte 1, n. 99.

10 Ibid., parte 2, n. ${ }^{\circ} 117$ et 118. 
deux précèdents patrons génois et n'en demande le remboursement qu'après le paiement du frêt ".

Voici donc un groupe de Musulmans, originaires de différents ports de l'Afrique du Nord et de Grenade qui, arrivés à Alexandrie d'Egypte, ont acheté de grandes quantités de lin et tentent de le vendre d'abord en Orient, puis le rembarquent vers Tunis: trafic aléatoire, risqué, aux conditions mal connues par avance mais qui, en l'occurence, emprunte le bâtiment génois qui se présente au bon moment et dont le patron offre de bonnes conditions. Finalement, fort embarrassés par leurs dettes et leurs sacs invendus, c'est un prêt d'argent qu'ils négocient auprès de ce Génois qui tient ici un autre rôle que celui de simple navigant-transporteur.

Le 20 juin 1457, Franchino de Valbona, habitant un petit bourg de la banlieue orientale de Gênes, et Andrea Boccario de Sturla, viennent témoigner, à la demande de Vinciguerra de Vivaldi, patron d'un bâtiment; ce dernier a été capturé l'été précédent, aux alentours de l'île de Malte in contractibus mate et goxii prope Siciliam) par une flotte de six galères pontificales commandée par l'archevêque de Tarragone; on lui a pris tout son chargement, ne lui laissant que soixante doubles, pièces d'or, pour son retour à Gênes. Les deux témoins ont été marins sur ce navire, qui avait chargé à Tunis des grains, blé et orge, pour Tripoli; le patron avait toûché 600 doubles de Tripoli de frêt et il lui en était resté 400 après avoir payé son équipage. À Tripoli, il avait entassé dans les cales de la nef différentes marchandises "pour sa Majesté le roi de Tunis»: des balles de laine, des cuirs, un tonneau de coloquinte (?), des nattes de roseaux tressés, des tonnelets d'eau de rose; était monté à bord un marchand maure, cum tribus capitibus maurorum, deux hommes et une femme. Bel exemple de ces trafics que condamnait la Papauté, ravitaillement en vivres, transports d'esclaves pour le compte de Musulmans; trafics qui, on le voit, s'exposaient tout de même à de cruels déboires. Vivaldi veut faire estimer ses pertes; il y parvient mais l'on ne voit pas comment il songe à récupérer l'argent ainsi confisqué ${ }^{12}$.

En avril 1466, il s'agit d'un autre patron, Giovanni Antonio Botto, qui fait reconnaître que l'esclave nommé Giacomino lui appartient bien; il l'a acheté à des Turcs qui, à Oran, étaient montés à son bord pour vingt doubles, pièces d'or; les vingt doubles furent ensuite déduits, au moment des comptes, des frêts que les Turcs devaient lui payer ${ }^{13}$.

Les formes des actes, leur répétition, montrent qu'il s'agit en somme de circonstances ordinaires, $d$ 'affaires conduites comme une routine: prendre à

$"$ Ibid., n. ${ }^{\circ} 119$.

12 ASG, Notaio Risso Baromeo, filza 5, n. 178.

13 ASG, Notaio Giovanni Vultabio; filza 1, non numérotée, acte du 2 avril 1466. 
bord des Maures est habitude et source de profits. Écoutons Anselme Adorno, parti de Gênes pour un pèlerinage en Terre Sainte et qui, ayant d'abord abordé à Tunis, poursuit au hasard des passages sa route vers l'Orient: «le 15 juin (1470), nous sommes montés sur un grand nanvire génois... dont le patron était Cosmo de Nigrono. Sur ce bâteau, se trouvaient une centaine de Maures, hommes et femmes; certains étaient des marchands qui avaient avec eux un chargement d'huile; d'autres, des pèlerins qui se rendaient à La Mecque; il y avait aussi des Juifs. On fêtait sur ce navires trois jours par semaine" ${ }^{14}$.

Gênes connaît certes, dans le Maghreb, des difficultés. Quelques conflits éclatent: imprudences ou actes de piraterie isolés. Mais ils ne semblent pas prendre des dimensions considérables; ils ne s'enflent pas, ne s'éternisent pas. Les captures dans les ports ou en haute mer, les razzias sur les côtes, les araisonnements de petites barques mal défendues ou les confiscations abusives des biens restent plutôt l'exception. C'est à Marseille et non à Gênes que fut créé autrefois, en 1198, l'ordre de la Très Sainte Trinité pour le rachat des captifs en pays musulmans; et à Barcelone celui de Notre-Dame-de-la-Merci, en $1218^{13}$. Ces ordres s' implantent très largement en Aragon et en Castille; on trouve des Mercédaires à Montpellier et même une maison des Trinitaires à Paris, là où avait étudié leur fondateur, Jean de Matha. Mais leur activité à Gênes semble plus limitée, au $\mathrm{XV}^{\mathrm{em}}$ siècle du moins; à cette époque, à lire les actes notariés, les accords pour racheter des Génois prisonniers n'apparaissent que très rarement ou pas du tout.

Les autorités génoises n'ont pas non plus à se préoccuper des entrepises de leurs patrons, à sévir constamment contre corsaires ou pirates de profession toujours à l'affût d'une prise, si médiocre soit-elle. Rien de comparable ici à ces continuelles récriminations, condamnations et suppliques qui tiennent en alerte le roi d'Aragon, obligé de punir ses sujets des Baléares et de Catalogne qui, sans cesse, compromettent les paix et les trèves, remettent tout en question ${ }^{16}$.

Pour les Génois, la seule affaire vraîment désagréable surgit en 1445 , avec le roi musulman de Grenade: dommages causés par des Génois à des gens de Malaga, confiscation des biens et créances de tous les Génois dans le royaume, institution d'une gabelle spéciale pour les dédommager; les échanges ne de-

14 J. HeERS et G. DE GROER, L'itinéraire, (cité supra), p. 141.

15 J. W. BRODMAN, The Trinitarian and Mercedarian Orders: A Study of religious redemptionis in the thirteenth century (Thèse. P.H.D. University of Virginia, 1974), Xerox University Microfilms, Londres, 1980.

${ }_{16} \mathrm{CH}$. E. DUFOURCQ, Catalogue chronologique et analytique du registre 1389 de la Chancellerie de la Couronne d'Aragon intitulé "Guerre Sairacenerorum 1367-1386", Barcelone, 1974. 
vaient reprendre qu'après de longues négociations ". Mais ce ne fut que représailles financières et absolument pas un conflit de sang: l'on ne comptait ni tués ni prisonniers. Aussitôt après, les trafics retrouvent un niveau très élevé et la Société génoise «du sucre et des fruits de Grenade» s'assure à nouveau de confortables bénefices. Almeria et Malaga demeurent d'importantes escales sur les grandes routes des nefs génoises. On peut même penser que les activités, les capitaux engagés, les intérêts de ces compagnies de commerce et des grandes familles de négociants ont, dans une certaine mesure, aidé ce royaume de Grenade à résister plus longtemps aux attaques des Castillans. Une lettre adressée de Malaga à Gênes, en 1482, ne fait état que d'une seule inquiétude: celle de voir s'avancer trop vite et trop souvent les armées des rois catholiques; cela ne vaut rien pour les affaires et peut compromettre une situation somme toute profitable ${ }^{18}$.

Si bien, qu'au total, les rapports avec les Musulmans d'Occident paraissent relativemente stables, favorables aux échanges commerciaux. Ni Gênes, ni les ports de ses Rivières ne se livrent à la chasse aux captifs. L'important est ici que, comme dans toute l'Italie du centre et du Nord, les esclaves d'origine africaine, les Musulmans en particulier, y son relativement rares. Ils ne forment qu'une très petite partie de la population servile. Pour la période comprise entre 1450 et 1470, Domenico Gioffré ne dénombre que 12 esclaves musulmans d'Occident, contre 69 Tatares, 157 Russes, 149 Circassiens et 58 Abkhazes (des vallées du Caucase) ${ }^{19}$. Le plus souvent d'ailleurs, ces hommes ou ces femmes ne font dans la ville qu'un court séjour, le temps que leur maître de l'heure les revende à un Catalan de passage ${ }^{20}$. De ce point de vue, en effet, Ligurie et Catalogne présentent des attitudes différentes: à Gênes, les esclaves sont, dans leur très grande majorité (environ $80 \%$ ), des femmes employées aux travaux domestiques et l'on ne garde pas volontiers chez soi, pour vaquer au ménage et surveiller les enfants, ces femmes d'Afrique qui refusent toute assimilation, refusent surtout le baptême, n'apprennent pas la langue, restent fidèles à leur moeurs. Les Musulmanes qui, signe intéressant, gardent toujours

1 G. AIRALDI, Genova e Spagna nel Secolo XV. Il "Liber damnificatorum in regno Granate. 1452», Gênes, 1966; J. HEERS, Le royaume de Grenade et la politique marchande de Gênes en Occident, in «Le Moyen Age» (1957), pp. 87-121.

18 ASG, Notaio Girolamo Ventimiglia, filza 1, acte n. ${ }^{\circ} 1$. 29.

19 D. GIOFFRE, Il mercato degli schiavi a Genova nel secolo XV, Gênes, 1971, p.

${ }^{20}$ Ventes à de petits négociants, à des commis, à des membres de la colonie catalane dispersés dans la ville, qui négocient les sacs de laine de leurs compatriotes et, volontiers, investissent cet argent dans l'achat d'esclaves que les Génois ne désirent pas garder. Cf. par ex.: ASG, Notaio Tommaso Duracino, filza 6, n. 27 (le 15 janvier 1461) et n..$^{\circ} 70$, Notaio Paolo de Recro, filza 1, n. ${ }^{\circ} 160$ (le 8 février 1452). 
leurs noms d'origine, ne peuvent convenir et cette désaffection, la préférence pour la traîte orientale, évite nombre de courses et de rapts.

Pour ce qui est des marchands maures, en tous cas, les hommes de bon rang, prisonniers par mauvais hasard, le rachat et le retour dans leur pays se négocient dans de bonnes conditions. Témoins ces trois contrats notariés successifs qui réflètent un parfait climat, une sorte de sollicitude. Tout se règle entre hommes d'affaires, au mieux. En août 1454, deux Maures, l'un «comprenant la langue génoise», l'autre «ne la comprenant pas mais se fiant en son compagnon comme interprète», se présentent devant le notaire ${ }^{21}$ et déclarent solennellement qu'ils se trouvent "dans le dominium et servitude de Giovanni Raibaldo, marchand génois qui les a achetés ou rachetés des mais de leurs ennemis", ainsi d'ailleurs que deux autres Musulmans qui n'apparaissent pas ce jour là ${ }^{22}$. Les deux hommes rachètent leur liberté, sont sur le point de s'embarquer sur le navire de Demetrio Cattaneo, et promettent de payer chacun, dans les vingt jours qui suivront leur arrivée à Tunis, «ou tout autre lieu de Barbarie», seize doubles et demi ainsi qu'une pièce de bocassin, étoffe de Tunis; paiement à effectuer dans les mains de Martino Raibaldo, frère de Giovanni, qui se trouve donc sur place. Quelques jours plus tard, ils apprennent qu'un autre bâtiment se prépare à lever l'ancre pour l'Afrique du Nord, celui de Baptista Salvaigo, navire tout neuf et plus grand que le précédent; ils se décident pour ce dernier et l'on refait le contrat ${ }^{23}$.

Ces conditions favorisent le trafic génois sur le littoral maghrébin. Face à la Provence et à la Catalogne, qui gardent, chez les citadins, de nombreux esclaves maures pour les travaux artisanaux, et lancent évidemmennt leurs navires à la chasse aux captifs et à la poursuite des fugitifs, Gênes, en place depuis fort longtemps, attentive à promouvoir en Méditerranée occidentale une "stratégie pour un empire» "24, l'emporte aisément sur tous ses rivaux.

Les Vénitiens passent au Maghreb généralement sans s'attarder. Preuve en est la façon dont les sociétés anonymes génoises contrôlent très régulièrement les pêcheries de corail de Marsacarès, alors que, sans conteste possible, ce marché du corail et les exportations vers l'Orient musulman ou byzantin sont, pour une bonne part, dans les mains de ces mêmes Vénitiens.

Les voyages génois "per costeriam» impliquent au contraire de nom-

${ }^{21}$ ASG, Notaio Risso Barameo, filza 3, parte 2, n. ${ }^{\circ} 138.139$.

${ }^{22}$ Ces Raibaldo, Giovanni et Martino, personnages bien modestes dans le milieu des hommes d'affaires génois, semblent donc directement intéressés par ce genre d'opérations: achats et reventes ou rachats de liberté des captifs musulmans.

${ }^{23}$ ASG, Notaio Risso Baromeo, filza 3, parte 2, n. 140.

${ }_{24}$ Cf. G. JeHEL, Les Génois et la Méditerranée occidentale. Ébauche d'une stratégie pour un empire (fin du XIP-début du XIV siècles), Thèse de Doctorat, Université de Paris-Sorbonne, 1987. 
breaux arrêts, une sorte d'éparpillement des activités, jusqu'en de médiocres escales, accidentelles et liées aux occasions; escales dont on tait même le nom mais que la formule générale "faire la côte» recouvre.

Pour les principaux ports, retenons Tunis, bien sûr, mais aussi Bône et Bougie, également Stora et, vers l'Ouest, Oran et Honein: chargements de grains et de laine, établissements sur place de commis ou de facteurs.

A Stora, par exemple, partium barbarie qui locus est carigatorium navis in loco Constantine ${ }^{25}$, en 1460, un marchand génois, Quilico Imperiale, était installé à demeure et possédait aussi une maison à Constantine ${ }^{26}$. Dans ce même port, les Génois avaient en permanence plusieurs facteurs, tels Oliviero di Negro en $1457^{27}$ et ceux qui représentaient, la même année, la société d'Emmanuele de Grimaldi et Benedetto di Negro, acheteurs de blés ${ }^{28}$.

Ainsi s'était tout naturellement établi un négoce très routinier, contrôle, pris en charge par ces représentants implantés dans quelques villes nordafricaines, en liaisons étroites certainement avec les marchés ruraux: prospections, achats à des intermédiaires, acheminements vers la côte, entrepôt dans la maison même du Génois ou à la douane.

Cependant, un autre type de transactions et d'exploitations de ces marchés indigènes mettait en jeu d'autres structures, plus simples et plus directes. C'était alors le patron du navire ou l'un de ses familiers, son scribe parfois, qui, outre le souci, non négligeable dans ces parages, de la navigation, se chargeaient de vendre ou d'acheter directement aux marchands maures résidant dans le port ou venus de l'arrière-pays, alertés par la nouvelle d'un passage maritime. Le 2 juillet 1456, Domenico de Malta, olim famulus nobilis Oberti Suarzafici patroni unius navi témoigne: en novembre 1455 (... vel circa...), à Stora, le scribe de ce navire, Rafaele de Monterosso, lui a donné, à lui Domenico, une certaine quantité d'argent pour acheter des grains dans le port; de plus, il sait que ce Rafaele a, sur place, échangé avec Ali Leoneymi (?), maure, une canne et demie de drap vert contre sept caffissi de grains ${ }^{29}$.

Par une chance exceptionnelle, un registre consigné aux officiers de la gabelle sur les transports maritimes, que l'on appelle les carati maris, permet de suivre de très près l'un de ces voyages "per costeriam» tout au long du periple ${ }^{30}$. Ces carati maris étaient régulièrement mis aux enchères et affermés à

25 ASG, Notaio Oberto Foglietta, filza 9, n. 411.

${ }^{26}$ ASG, Notaio Tommaso Duracino, filza $7, \mathrm{n} .^{\circ} 72$, cuirs transportés à Collo et à Stora.

27 ASG, Notaio Antonio di Fazio, filza 18, n. 577.

${ }^{28}$ ASG, Notaii Giudiziali, not. Benedetto Pilosio, le 10 février 1457.

${ }^{29}$ ASG, Notaio Tommaso Duracino, filza 2, parte 2, n. ${ }^{\circ} 175$, le 2 juillet 1486. 1552.

${ }^{30}$ ASG, Archivio di San Giorgio, sala 38/54. Caratorum Vetterum, anno 1445, n. 
des financiers qui appliquaient les taux prévus par les status, mais pouvaient accorder toutes sortes de privilèges, conditions particulières, exemptions ou rabais. Le plus claire de leurs efforts visaient, par ailleurs, à démasquer les fraudeurs, à les confondre par des témoignages, à récupérer une partie de l'argent ainsi perdu. A la différence de Venise, aucun agent ni de l'État, ni de la Casa di San Giorgio, ni des fermiers de l'impôt ne surveillait le patron à son bord. Tout reposait sur ses déclarations et sur celles des marchands. Lorsque l'abus paraissait trop manifeste, l'on interrogeait diverses personnes présentes ou informées, l'on exigeait les livres de bord et les registres comptables des particuliers pour les examiner.

C'est, dans ces circonstances, que, pour l'an 1445, les fermiers des carati obtenaient de Filippo di Negrono, patron d'un bâtiment, la copie de toutes ses écritures de bord; cette copia unius manualis navis Filippi de Nigrono fut finalement présentée en avril 1448, plus de trois ans plus tarde...

Aucun autre document de ce genre ne nous est conservé pour cette époque. Tous les livres de bord, les registres de frêt en particulier qui permettent de suivre les opérations et les trafics au jour le jour, tous ont disparu; sauf, précisément celui-ci, confisqué par les hommes de l'impôt et depuis lors gardé dans leurs archives.

En fait, le registre comprend, en un même et seul volume, plusieurs parties distinctes, mais qui se complètent l'une l'autre; de toutes façons, l'itinéraire se lit clairement. Le navire est parti d'Angleterre (vraisemblabement de Southampton) et atteint Cadix; de là, il toûche Malaga, puis Honein, puis Oran et suit la côte d'Afrique jusqu'à Tunis; et, de là, se rend a Chio. Filippo di Nigrono a noté d'abord ce qui est chargé au départ (nota quod babeo in nave, de racionis...). Ces indications très nombreuses et très complètes font état de la date du chargement, du propiétaire des marchandises, de leur nature (surtout de beaux draps anglais, draps «larges» en particulier, dits "de Ludlow»), du nombre de balles ou de pièces, de la marque apposée sur l'emballage. Viennent ensuite, dans les moindres détails, les instructions pour les livraisons ou même les ventes de ces draps: où? à qui? à quel prix? Parfois l'on donne pour consigne non de vendre mais d'échanger contre certains produits; enfin, il est dit à qui consigner l'argent de ces ventes et commment le faire éventuellement parvenir à Gênes. Ceci paraît fort complexe, précis, en prévoyant toujours des solutions de rechange: si tel ou tel marchand ou commis n'a pas voulu de nos draps à Malaga, proposez-les «per costeriam», puis à un négociant de Tunis et enfin à Chio.

Ainsi, par exemple, les premières mentions concernent-elles deux balles de draps, l'une de 46 pièces, l'autre de 23, appartenant pour deux tiers à Agostino Salvaigo et pour l'autre à Francesco di Nigrono;" le registre précise, de plus, quas ballas conducere babeo secretas, mention que l'on retrouve, au long des 
folios, à plusieurs reprises. Le profit de ces deux balles, à livrer à Malaga ou à vendre «per costeriam» ou à Tunis, est à envoyer à Gênes sous forme d'or ou de soie grège.

La deuxième partie du registre est consacrée au relevé des frêts, classés par lieux de déchargements, évalués en monnaies des pays (doubles de Castille, de Grenade et diverses doubles du Maghreb), payés donc par ceux qui ont reçu et accepté la marchandise. Cette disposition souligne nettement le caractère aléatoire de ces transports: aucun tarif fixé à l'avance, aucun engagement de paiement au départ; le patron ne toûche ses frêts, ne peut payer ses marins et assurer la course de son bâtiment qu'au fur et à mesure des consignations et des ventes, et donc de la progression du voyage. $\grave{A}$ ces ventes, il se trouve ainsi directement intéressé, s'employant de son mieux à placer les balles de draps de très belles qualités, qui représentent de véritables fortunes; il sollicite lui-même les clients, fait valoir les marchandises et, à l'intérieur de certaines limites certes, joue sur les prix: situation originale, que l'on ne retrouve nulle part ailleurs sur aucune route maritime parcourue par ces nefs de Gênes.

Une dernière partie du livre porte le titre rauba vendita per costeriam et compte plus d'une dizaine de feuillets. C'est le relevé de tous les draps, de leur qualité et de leurs couleurs, du nombre de pièces, vendus directement par le patton à des «maures» dans les ports, au passage. Ici, l'on ne compte plus en balles mais en pièces et même en coupons dont on indique la longueur exacte, à la "paume» près: commerce de détail, de petit détail même. Malheureusement, les lieux et les clients ne sont pas toujours indiqués car il est net que le patron assume alors pour ces ventes-là toute responsabilité: les marchands doivent lui faire confiance. On cite Bougie à plusieurs reprises; on dit que des draps ont été vendus "au roi de Bougie» ou "au sérénissime roi de Tunis»; d'autres sont simplement échangés. Et ceci entraîne des visites, sinon dans tous les cas aux palais des souverains, du moins à des négociants accrédités auprès d'eux.

Au-delà de l'anecdote, du film de la navigation elle-même, de l'organisation des trafics, des variations de prix et de modes, ce registre met en pleine lumière des pratiques étroitement conditionnées par un contexte particulier et confirme ainsi ce que laissaient entrevoir quelques actes notariés, eux plus précis mais ponctuels et dispersés. Il paraît certain que les marchands et patrons génois ont su adapter la navigation de leurs énormes nefs lancées sur la ligne maritime la plus longue du monde (d'Angleterre à Chio) à la prospection de marchés très dispersés et d'accès difficiles. Les dimensions de ces navires, leur puissance et leur armement, les rendaient pratiquement invulnérables à toute attaque de mer ou de terre, tant dans les ports qu'au large. La nef, très haute sur l'eau, véritable forteresse, peut défier toute une flottille de pirates; c'est un refuge où les hommes, une centaine de marins, se sentent parfaitement à l'abri. 
Aussi ces négoces se poursuivaient-ils impunément, mettant en jeu des sommes considérables, dans une sorte de tranquillité loin d'une quelconque protection "politique» des souverains ou de leurs officiers... ce que ne pouvaient naturellement pas se permettre d'autres bâtiments de moindres tonnages, barques ou petites nefs, plus exposées aux attaques des ennemis. Ces pratiques s'étaient peu à peu imposées face aux nécessités des transports de masse. Ces nefs génoises, portant plusieurs centaines de tonnes métriques, avaient été lancées pour porter les grains, les toneaux de vin, les balles de pastel et, surtout, l'alun d'Orient: capables d'emporter des cargaisons considérables, montées par des équipages relativement réduits, elles permettaient de n'exiger que des frêts somme toute modestes et de faibles primes d'assurance pour tous ces produits lourds.

Au XV ${ }^{\text {ent }}$ siècle, le trafic d'Orient n'est plus du tout celui des "épices» légères et précieuses; mais celui, essentiellement, de l'alun, produit bon marché; pour les transports vers l'Occident, les grosses nefs conviennent parfaitement. La même recherche d'une diminution des frais a conduit à choisir des itinéraires plus courts, sans remonter vers le Nord jusqu'à Gênes et, surtout, sans rupture par un arrêt prolongé soumis à un changement de navire, à des vérifications douanières sourcilleuses, engendrant d'autres retards, voire même des amendes. D'où le choix de cette pratique "per costeriam».

La comparaison entre les grandes «nations» maritimes d'Italie s'impose d'une manière particulièrement nette, en ce domaine. Pour Venise et pour Florence, un produit d'Orient destiné à la Flande ou à l'Angleterre est d'abord chargé sur un navire, une galée généralement, qui s'agrège à un convoi et gagne ou la Cité des lagunes, tout au fond de l'Adriatique, ou Porto Pisano: perte de temps appréciable. Arrivé là, le marchand doit faire débarquer, entreposer, attendre (des jours ? des semaines?) l'annonce d'un convoi pour l'Occident et, enfin, faire charger sur une autre galée qui effectue un trajet inutile, au sens contraire de l'aller. Pour Gênes, à l'inverse, tout allait très vite: l'alun de Phocée chargé à Chio ne transitait jamais par le port ligure.

Au retour, d'Angleterre vers le Levant, la nef, réputée à juste titre invincible, peut s'aventurer en vue des côtes du Maghred pour de longues investigations des marchés, portant des cargaisons de très grande valeur, de produits véritablement de luxe, sans craindre d'en perdre quoi que ce soit. Le patron du navire et les marchands à son bord visitent ainsi nombre de marchés, multiplient les occasions de clientèles. Ces opérations très diverses s'effectuent en dehors de tout contrôle de l'État génois ou même des fermiers des gabelles. Ce qui laisse au capitaine du bâtiment, à ses commis, aux marchands passagers, une grande part d'initiative. Les lignes, étapes et contacts, ne sont pas toutes tracées à l'avance. Chaque voyage se définit comme une aventure et donc, pour les hommes, par un enrichissement, un acquis d'expériences nouve- 
lles dont, peu de temps après, certains de ces hommes de mer tireront profit pour l'exploration de nouveaux marchés, de nouveaux mondes même.

\author{
JACQUES HEERS \\ Université de Paris-Sorbonne
}

\title{
RÉSUMÉ
}

Toutes les nations maritimes de la rive Nord de la Méditerranée s'intéressent au commerce avec le Maghreb et y exercent diverses activités. Mais toutes n'agissent pas de la même façon et la ville de Gênes semble, dans ce secteur, avoir suivi une politique très particulière.

Les trafics entre Gênes et le Maghreb bénéficiaient, au XVème siècle, d'une sorte de paix: peu d'actes de piraterie, peu de représailles, peu de captures d'esclaves; dans Gênes, les esclaves en provenance du Maghreb sont très rares et les marchands ou patrons de navires s'emploient volontiers aux rachats des captifs.

L'originalité du système de navigation réside dans les voyages que les notaires appellent "per costeriam»; c'est à dire, de longs pépriples maritimes qui, pour de très gros navires, vont directement d'Angleterre en Orient (ou réciproquement) en longeant la côte d'Afrique, sans remonter vers le Nord et faire escale à Gênes: économie de temps et d'argent. Pour ces voyages le patron de navire dispose d'une très grande initiative et assume de grandes responsabilités: navigation, chargements et déchargements des balles de marchandises (en particulier des draps englais), mais aussi et surtout ventes de ces produits. Ceci implique un nombre important d'escales sur le littoral du Maghreb (parfois au large d'une simple plage) et, aussi, la fréquentation de nombreux marchés ou de négociants musulmans. Ces trafics "per costeriam» sont de véritables aventures et les capitaines des navires y ont acquis une grande expérience des mers, des côtes, des populations étrangères à leur monde chrétien.

\section{SUMMARY}

All the seaboard nations of the Mediterranean North coast feel interest to trade with the Maghreb and do already realize activities over there. However, all of them do not act in the same way, and the city of Genova seems to follow a very special policy in this area.

The commerce between Genova and the Maghreb used, during the $\mathbf{X V}^{\text {th }}$ century, to benefit from a kind of peace: few acts of piracy, few reprisals, few slave captures: in Genova, the slaves coming from the Maghreb were extremely unfrequent, and the tradesmen and skippers usually bought back the captives. 
The originality of the navigation system lies in the trips that the notaries call "per costeriam». They are, in fact, long maritime voyages; the really big ships go straight from England to the Orient -or vice versa- bordering the African coast, without going back upwards to the North nor calling at Genova, which supposed a gain of time and money. For these trips, the skipper enjoys a very large margin of initiative and assumes important responsibilities: the navigation, the loading and unloading the bales of goods -specially English woollen cloth-, but also and above all, the selling of these products. This implies to call frequently at the Maghreb -sometimes on a mere large beach- and to stop at several markets and visit Muslim tradesmen. These trades "per costeriam" are real adventures and the captains acquire so a large and useful experience of the oceans, of the coasts and of the populations which are different from their Christian world. 